\title{
Harold S. Shapiro at KTH: some personal memories
}

\author{
Björn Gustafsson ${ }^{1}$ (B)
}

Received: 19 January 2022 / Accepted: 20 January 2022 / Published online: 8 February 2022

(c) The Author(s) 2022

\section{Abstract}

The author, who was a student and colleague of Harold S. Shapiro, gives some personal memories from Shapiro's time as a professor at KTH, Stockholm, 1972-2021.

After several research visits to Sweden in the 1960:s Harold S. Shapiro was in the early 1970:s offered a temporary professorship in mathematics at KTH, Stockholm. Harold had always admired, and been attracted by, the strong Swedish tradition in mathematical analysis, with world leading names such as Torsten Carleman, Arne Beurling, Lennart Carleson, and Lars Hörmander. In addition, he had fallen in love with, and married, a young Swedish woman, Karin Tegmark, in the 1960:s. So he must have been very happy for this offer from Stockholm.

At KTH two of the professors in the 1960:s were Göran Borg and Bo Kjellberg, both of them students of Beurling (like Carleson). By 1970, Göran Borg had been appointed dean of the entire school KTH, and Kjellberg spent much of his time on giving classes for engineering students. There were still some promising younger mathematicians around, but in principle there was a lack of a mathematics professor who really took on doctoral students and gave courses for them. There was also a competition with Stockholm university, where Jan-Erik Roos recently had become professor and had started a very ambitious program for doctoral students.

It was in this partial vacuum that Harold was recruited. And his start, in September 1972, was full-powered. That first academic year 1972/73 he gave a personally featured course based on Walter Rudin's book "Real and complex analysis". He actually started from chapter 10, on holomorphic functions, since basic real analysis was covered by other courses. Examination was by homework, problems that Harold in most cases had invented himself. Harold attracted immediately a substantial group of students for the course, and via the homework he could see who were good enough to become doctoral students and eventually write a thesis (Fig. 1).

The following academic years continued in the same style, but with new courses every year, and in a great variety of subjects: Fourier analysis, partial differential equations, functional analysis, Sobolev spaces, operator theory, approximation theory,

\footnotetext{
$凶$ Björn Gustafsson

gbjorn@kth.se

1 Royal Institute of Technology, Stockholm, Sweden
}

Birkhäuser 


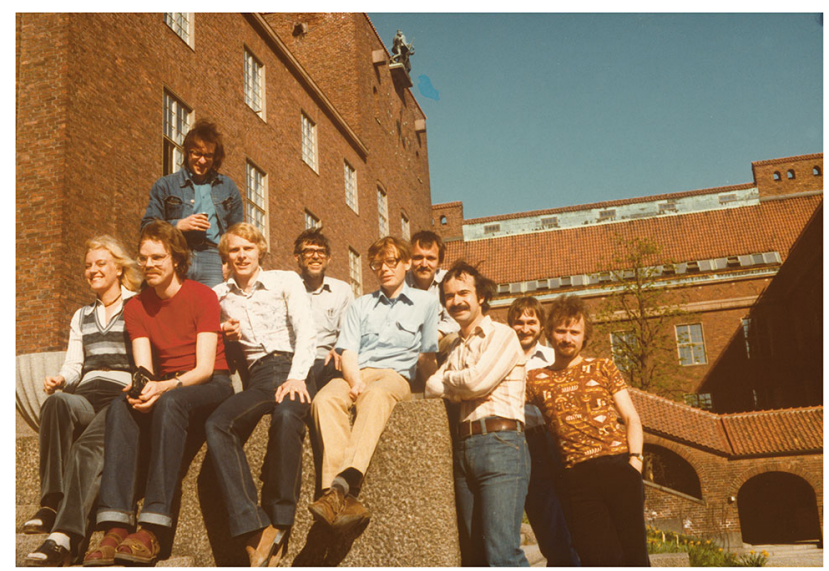

Fig. 1 Harold S. Shapiro with some doctoral students at campus of KTH, probably in spring of 1977. From left: Carina Ullemar, Roland Kjellander, Wojciech Abramczuk (standing), Anders Östrand, Harold Shapiro, Björn Gustafsson, Lars Svensson, Andrzej Szulkin, Mats Lindberg, Carl Engblom

geometric function theory, mathematical logic, number theory, topological analysis, and more. Later on also topics from applied mathematics, such as elasticity theory, were taken up.

A very special feature was Harold's love for problem solving. Much inspired by books of Polya and Szegö he started giving seminars and courses on problem solving, both for doctoral students and for engineering students. They became very popular, and the tradition was followed up by other teachers, in particular Lars Svensson.

Harold was a very good lecturer. He taught mainly doctoral courses and his presentations were always very well prepared and organically disposed. Usually there was only one class a week, two hours with a break, and he preferred to schedule this for Tuesday afternoon. This in order to have the full Monday plus Tuesday morning for preparations. The result was a manuscript with complete details. However he almost never used the manuscript in class, the only thing he possibly needed was a small sheet of paper kept in the breast pocket ("fusklapp" in Swedish) containing complicated formulas which could be difficult to remember by heart.

Besides the doctoral courses there were different kinds of seminars, general seminars for the whole department and other interested mathematicians, as well as specialized seminars within the research group. The latter were often followed up by small tea parties with informal mathematical discussions. Quite many research projects started from ideas which came up during these parties. Often Harold had made some simple observation, for example concerning the zero locus of a real polynomial of degree four in two variables. And then someone realized that this was a special case of something more general. And after that the snow ball had started rolling, and everything could eventually develop into a series of publications with many mathematicians involved. In some cases whole new research areas were created in this way. One could easily list tens of mathematical publications which are spin-off effects of Harold's tea parties. 
To mention just one (out of many possible) important contribution in Harold's scientific production I would pick his paper, with Dov Aharonov, from 1976 with title "Domains on which analytic functions satisfy quadrature identities", in J. Analyse Math., vol. 30, pp. 39-73. This paper is, for good reasons, much cited. In fact, it marks the starting point for a whole new research area, namely that of "quadrature domains", lying in the meeting point between complex analysis, potential theory and free boundary problems, the latter as being part of nonlinear PDE.

What makes Aharonov's and Shapiro's treatment of quadrature domains stand out, for example from Philip Davis' related but more numerically oriented studies of the Schwarz function, is that Aharonov-Shapiro start from very general assumptions. In a Lemma 2.3 in the mentioned paper the two authors assume just that a bounded domain (or even open set) satisfies a finite quadrature identity for integrable analytic functions and conclude from this that there exists a meromorphic function $S(z)$ in the domain which is continuous up to the boundary and satisfies $S(z)=\bar{z}$ there. The name "Schwarz function" for this $S(z)$ had already been used by Philip Davis, but AharonovShapiro called the function $h(z)$ (probably they became aware of Davis' work rather late in the writing process). The implication also goes in the other direction, the lemma is stated as an equivalence between four different statements. The proof is actually more or less straight-forward. But what is important is what comes next, namely their Theorems 2 and 3.

By using short and elementary, but clever, arguments the authors show in these two theorems that the boundary of any domain which admits a finite quadrature identity for integrable analytic function must be a subset of an algebraic curve. This step, from no assumption at all on the boundary to the very strong algebraicity, is very important. Having that global grip the further development of the theory is relatively easy. Nothing similar can be done for corresponding questions in higher dimensions (say finite point quadrature identities for harmonic functions), as far as we know. And even in two dimensions, if one relaxes the assumption on the support of the quadrature functional to be a compact set, in place of a finite set, complete statements of boundary regularity (real analyticity with certain kinds of singularities allowed) require proofs of considerable length (for example 35 pages in the first complete treatment, by Makoto Sakai in Acta Math. 1991).

The mentioned results on quadrature domains have been important for me personally, and the proof that the boundary has to be a subset of an algebraic curve has impressed not only me, but many mathematicians. To pick something more, I choose a general seminar that Harold gave at the department. Harold had a good relation to functional analysis, already since the time of his doctoral thesis, and one day he decided to give a seminar to present, in one stroke, full proofs of the three most important theorems in functional analysis: the Hahn-Banach theorem, the open mapping (or closed graph) theorem, and the Banach-Steinhaus theorem. And so he did, in an absolutely brilliant way, and without ever looking in his manuscript. This impressed me a lot.

Funding Open access funding provided by Royal Institute of Technology.

Data availability All data needed are contained in the manuscript. 


\section{Declarations}

Conflict of interest The author certifies that he has no affiliations with, or involvement in, any organization or entity with any financial interest or non-financial interest in the subject matter discussed in this manuscript.

Open Access This article is licensed under a Creative Commons Attribution 4.0 International License, which permits use, sharing, adaptation, distribution and reproduction in any medium or format, as long as you give appropriate credit to the original author(s) and the source, provide a link to the Creative Commons licence, and indicate if changes were made. The images or other third party material in this article are included in the article's Creative Commons licence, unless indicated otherwise in a credit line to the material. If material is not included in the article's Creative Commons licence and your intended use is not permitted by statutory regulation or exceeds the permitted use, you will need to obtain permission directly from the copyright holder. To view a copy of this licence, visit http://creativecommons.org/licenses/by/4.0/.

Publisher's Note Springer Nature remains neutral with regard to jurisdictional claims in published maps and institutional affiliations. 